\title{
SMOOTH PERTURBATIONS OF A FUNCTION WITH A SMOOTH LOCAL TIME ${ }^{1}$
}

\author{
BY
}

\author{
D. GEMAN AND J. HOROWITZ
}

\begin{abstract}
A real Borel function on [0, 1] has a local time if its occupation measure up to each time $t$ (equivalently: its increasing, equimeasurable rearrangement on $[0, t])$ is absolutely continuous; the local time $\alpha_{t}(x)$ is then the density. An inverse relationship exists between the smoothness of the local time in $(t, x)$ and that of the original function. The sum of a function with a smooth local time and a well-behaved (e.g. absolutely continuous) function is shown to have a local time, which inherits certain significant properties from the old local time, and for which an explicit formula is given. Finally, using a probabilistic approach, examples are given of functions having local times of prescribed smoothness.
\end{abstract}

1. Introduction. Let $X(t), 0 \leqslant t \leqslant 1$, be a real-valued Borel function, let $m$ be Lebesgue measure, and suppose the measure $\mu(\Gamma)=m\left(X^{-1}(\Gamma)\right)$ on the Borel sets of $\mathbf{R}$ is absolutely continuous; then, for each $t, \mu_{t}(\Gamma)=m\left([0, t] \cap X^{-1}(\Gamma)\right)$ is likewise absolutely continuous and the Radon-Nikodym derivative $\alpha_{t}(x)=$ $\mu_{t}(d x) / m(d x)$ is called the local time of $X$.

A function with a "smooth" local time is very irregular; for instance, if $\alpha_{t}(x)$ is simply jointly continuous, then $X$ is nowhere differentiable and has uncountable level sets. If we "perturb" $X(t)$ by a regular function, e.g. by adding an absolutely continuous function $Z(t)$, then we might expect $X(t)+Z(t)$ also to be irregular. We shall show that such perturbations do indeed preserve many significant features of $X(t)$ in the sense that the local time of the resulting function exists and inherits certain properties from $\alpha_{t}(x)$. Our attention will be focused on sums of functions, but the same methods apply to other types of perturbations and yield qualitatively similar results; an illustration is given in $§ 6$.

The following is an easily stated example of the type of result we can get; a stronger form is given in Theorem A, $\$ 3$.

TheOREM $\mathrm{A}^{\prime}$. Let $Z(t)$ be continuously differentiable and let $X(t)$ have a jointly continuous local time $\alpha_{t}(x)$ such that $x \mapsto \alpha_{t}(x)$ is absolutely continuous for each $t$, and $\alpha_{t}^{\prime}(x)=\partial \alpha_{t}(x) / \partial x$ is integrable on $[0,1] \times \mathbf{R}$; then $X(t)+Z(t)$ has a local time given by

$$
\gamma_{t}(x)=\alpha_{t}(x-Z(t))+\int_{0}^{t} \alpha_{s}^{\prime}(x-Z(s))(d Z / d s) d x .
$$

Received by the editors January $30,1980$.

AMS (MOS) subject classifications (1970). Primary 26A27, 60G17; Secondary 26A45, 28A15.

Key words and phrases. Occupation measures, local time, functions of a real variable, bounded variation, Gaussian process.

${ }^{1}$ This work partially supported by NSF Grant MCS 7606599. 
A heuristic proof of this theorem will be given in $\$ 5$.

We use a probabilistic approach to give examples of functions $X(t)$ which satisfy the hypotheses of the above theorem. Such functions are difficult to construct directly, so instead we produce a Gaussian process (really a family of such processes) almost every trajectory of which has the desired properties. These are the so-called index $\gamma$ processes; the exact definition, along with the proof of the following theorem, is given in $\$ 7$.

TheOREM D. Almost every trajectory $X_{t}$ of an index $\gamma$ process, with $\gamma<\frac{1}{2}$, has a jointly continuous local time $\alpha_{t}(x)$, which has a jointly continuous (space) derivative $\alpha_{t}^{\prime}(x)$.

Since $X_{t}$ is continuous (with probability 1 ) in such cases, $\alpha_{t}(x)$ is supported on $[0,1] \times X[0,1]$, and therefore $\alpha_{t}^{\prime}(x)$ will be integrable on $[0,1] \times \mathbf{R}$. We remark that arbitrary smoothness in the "space" variable $x$ may be achieved by further reducing $\gamma$.

Although smoothness of $\alpha_{t}(x)$ in the space variable $x$ enters into the above theorems, it is the behavior of $\alpha$ in the time variable $t$ which accounts for much of the irregular behavior of $X$. Let

$$
\omega_{\alpha}(h)=\sup _{t, x}\left|\alpha_{t+h}(x)-\alpha_{t}(x)\right| .
$$

Some consequences of the joint continuity of $\alpha_{t}(x)$ were already mentioned, but finer properties are controlled by $\omega_{\alpha}(h)$. For instance, if $\omega_{\alpha}(h)=O\left(|h|^{\beta}\right), h \rightarrow 0$, for $0<\beta<1$, then $X$ cannot satisfy a local Hölder condition of order $>1-\beta$ at any point; and, for a.e. $t$, the Hausdorff dimension of $\{s: X(s)=X(t)\}$ is $\geqslant \beta$. A complete discussion of these ideas, which originate with S. Berman, will be found in our survey article [5]. The behavior of the perturbed local time is now described in the following way. It will be clear, after $\$ 2$ (or see [5]) that, for a.e. $x, \alpha_{t}(x)$ is a monotone increasing singular function of $t$, so there is little harm in assuming $\omega_{\alpha}(h) / h \geqslant \eta>0$ for $h$ sufficiently small. Indeed, $\partial \alpha_{t}(x) / \partial t=\infty$ on an uncountable set, typically. We then have

COROLLARY. Under the above assumption and the hypotheses of the preceding theorem,

$$
\omega_{\gamma}(h)=O\left(\omega_{\alpha}(h)\right)
$$

The proof follows easily from (1) and is omitted.

Part of the motivation for the present work lies in the following theorem of Meyer [7, p. 368]: let $Z_{t}$ be a random process with almost all trajectories of bounded variation, adapted to the canonical $\sigma$-fields of a Brownian motion $X_{t}$; then, a.s., $X_{t}+Z_{t}$ has a local time. Though the proof involves the heavy machinery of stochastic integrals, the statement itself suggests a purely real-variable explanation in the spirit of the above discussion: indeed, a.s. the Brownian motion has a jointly continuous local time, so the addition of $Z_{t}$ should not appreciably dampen the oscillations of $X_{t}$. Theorem A below may be regarded as an attenuated real-variable analogue of Meyer's theorem. We may mention also the papers [2] and [11] which 
deal with the cardinality of the range of $X(t)+Z(t)$, where $Z(t)$ is continuous and of bounded variation and $X(t)$ is a Darboux function which maps every interval onto $\mathbf{R}$.

2. Preliminaries. We will give the results for vector-valued functions of a real variable, noting that the existence of a local time for such functions implies much wilder behavior than in the real-valued case.

Let $X:[0,1] \rightarrow \mathbf{R}^{d}$ be a Borel function, $\mathscr{B}_{d}$ and $m_{d}$ the Borel sets and Lebesgue measure in $\mathbf{R}^{d}$ (we drop sub- and superscripts when $d=1$ ), and $\mathscr{B}([0,1]$ ) the Borel sets in the unit interval. The occupation measure of $X$ relative to $A \in \Re([0,1])$ is

$$
\mu_{A}(\Gamma)=m\left(A \cap X^{-1}(\Gamma)\right), \quad \Gamma \in \mathscr{B}_{d} .
$$

We write simply $\mu_{t}$ when $A=[0, t]$. If

$$
\mu_{1} \ll m_{d}
$$

then $\mu_{A} \ll m_{d}$ for each $A$, and $\mu_{A}$ has a disintegration

$$
\mu_{A}(\Gamma)=\int_{\Gamma} \alpha(x, A) d x .
$$

We call $\alpha$ the occupation density of $X$. It is known that $\alpha$ may be chosen to be a kernel, meaning that $\alpha(\cdot, A)$ is a Borel function for each $A$, and $\alpha(x, \cdot)$ is a finite measure on $\mathscr{B}([0,1])$ for each $x \in \mathbf{R}^{d}$. It will always be assumed that such a choice has been made. The function $\alpha_{t}(x)=\alpha(x,[0, t])$ is the one introduced (in the case $d=1)$ in $\$ 1$; it is called the local time.

An important formula which will be needed later is this: under condition (*),

$$
\int_{0}^{1} g(s, X(s)) d s=\int_{\mathbf{R}^{d}} \int_{0}^{1} g(s, x) \alpha(x, d s) d x
$$

for any nonnegative or bounded, $\mathscr{B}([0,1]) \otimes \mathscr{B}_{d}$-measurable function $g$. When $g(s, x)=I_{A}(s) I_{\Gamma}(x)$, (5) reduces to (4), and, for general $g,(5)$ is obtained by a monotone class argument.

Taking $g(s, x)=1$ if $X(s) \neq x,=0$ otherwise, we find that, for a.e. $x$, the measure $\alpha(x, d s)$ is carried by $\{s: X(s)=x\}$, which is always a set of Lebesgue measure 0 under $(*)$. Thus $\alpha_{t}(x)$ is a singular function of $t$, as mentioned in $\S 1$.

For more background material, see [5], where there is a discussion of the history and utility of occupation densities, and where some of the present results were announced.

3. Main results. We will use the abbreviations (BV) and (AC) for "bounded variation" and "absolutely continuous", respectively; phrases such as " $Z(t)$ is (BV)" are then self-explanatory. Let $Z:[0,1] \rightarrow \mathbf{R}^{d}$ be of bounded variation. The definition in the vector-valued case is similar to that in the real-valued case; we refer to Federer [3, p. 109] for details. Writing out the coordinates of $Z(t)$, it is easily seen that $Z(t)=\left(Z_{1}(t), \ldots, Z_{d}(t)\right)$ is $(B V)$ iff each $Z_{i}(t)$ is $(B V)$ in the usual sense.

The function $Z(t+)$ is right continuous, has left limits, and agrees with $Z$ off a countable set; thus $X(t)+Z(t+)$ and $X(t)+Z(t)$ have the same occupation measure, so that we may, and do, assume $Z$ is right continuous. 
Let $\Delta Z(t)=Z(t)-Z(t-)$ be the jump at $t$. We have

$$
\sum_{t \in[0,1]}|\Delta Z(t)|<\infty,
$$

where $\|$ indicates the usual norm in $\mathbf{R}^{d}$. Denote by $\nu_{Z}(z, B)$ the cardinality $(<\infty)$ of the set $B \cap Z^{-1}(z)$. This is called the Banach indicatrix of $Z$. It is easy to check that $\nu_{Z}$ is a kernel on $\mathbf{R}^{d} \times \mathscr{B}([0,1])$, at least if $\mathbf{R}^{d}$ is endowed with the $\sigma$-field of universally measurable sets.

We are going to introduce in $\$ 4$ a scalar variation measure $v_{z}$ and a vector variation measure $V_{Z}$ on $\Re([0,1])$. The continuous part of $v_{Z}$ is given by [3, p. 177]

$$
\psi_{Z}(B)=\int_{\mathbf{R}^{d}} \nu_{Z}(z, B) H^{1}(d z), \quad B \in \mathscr{B}([0,1]),
$$

$H^{1}$ being the 1-dimensional Hausdorff measure on $\mathbf{R}^{d}$ (= Lebesgue measure if $d=1$ ). The continuous part of $V_{Z}$ will be a vector-valued measure $\Psi_{Z}$. Here is the main result.

Theorem A. Suppose $X(t)$ has a jointly continuous local time $\alpha_{t}(x), Z(t)$ is $(B V)$, and $\alpha$ is subject to the following:

(a) for $\psi_{z}$-almost every $s, \alpha_{s}\left(x_{1}, \ldots, x_{d}\right)$ is absolutely continuous with respect to each variable separately,

(b) $\left|\nabla \alpha_{s}(x)\right|$ is in $L^{1}\left(\psi_{Z} \times m_{d}\right)$ (the gradient is taken with respect to $x=$ $\left(x_{1}, \ldots, x_{d}\right)$ ),

(c) $\sum_{s}\left|\alpha_{s}(x-Z(s))-\alpha_{s}(x-Z(s-))\right|$ is in $L^{1}\left(\mathbf{R}^{d}\right)$.

Then $X(t)+Z(t)$ has a local time; it is given by

$$
\begin{aligned}
\gamma_{t}(x)= & \alpha_{t}(x-Z(t))+\int_{0}^{t} \nabla \alpha_{s}(x-Z(s)) \cdot \Psi_{Z}(d s) \\
& -\sum_{s<t}\left[\alpha_{s}(x-Z(s))-\alpha_{s}(x-Z(s-))\right] .
\end{aligned}
$$

Moreover, $\gamma .(x)$ is continuous for a.e. $x$.

The dot product integral in (8) is an obvious shorthand for a sum of numerical integrals. It becomes apparent, after some decoding, that Theorem $A^{\prime}$ in the introduction is a special case of Theorem A. Here are some further variants.

COROllary. Suppose $X(t)$ is bounded, (a) holds for every $s$, and $\left|\nabla \alpha_{s}(x)\right|$ is bounded; then $X(t)+Z(t)$ has a local time and (8) holds for every $Z$ of $(B V)$.

The boundedness of $X$ guarantees that $\alpha_{s}(x)$ and $\left|\nabla \alpha_{s}(x)\right|$ both vanish for $|x|$ sufficiently large, so (b) holds; and then (6) and

$$
\left|\alpha_{s}(x-Z(s))-\alpha_{s}(x-Z(s-))\right| \leqslant\|\nabla \alpha\|_{\infty}|\Delta Z(s)| I_{\{x:|x|<k\}},
$$

where $k$ is sufficiently large, show that (c) holds. Hence the corollary is proven. Another consequence of Theorem A goes in a different direction.

THEOREM B. Suppose (a) holds and $\left|\nabla \alpha_{s}(x)\right|$ is Lebesgue integrable on $[0,1] \times \mathbf{R}^{d}$; then, if $Z$ is approximately differentiable a.e., $X(t)+Z(t)$ has a local time. 
In this case a formula like (8) cannot be expected to hold. The approximate derivative is defined to be the so-called approximate limit of the usual difference quotient; see Saks [10], Federer [3], and [4]. A proof of Theorem B can be easily manufactured out of Theorem A and Federer [3, 3.1.16]; we omit the details. Part of the interest of Theorem B is in the context of stochastic processes, where it is relatively easy to prove, e.g., measurability properties for the approximate derivative of a process, while being difficult or impossible for the true derivative; see [4].

4. Technicalities on functions of bounded variation. We begin with various technical facts about (BV) functions which are somewhat different from those appearing elsewhere, and which are needed for the proof of Theorem A.

Let $v(t)$ be a right continuous, nondecreasing function on $[0,1]$. The induced measure on $\mathscr{B}([0,1])$ is also denoted $v$, and we write $v(A)$ for the $v$-measure of the set $A$, but $v[A]$ for the image of $A$ by the function $v(t)$. The continuous (i.e. atomless) component of $v$ will be denoted by $p$, referring either to the function or the measure. We now show that

$$
m(A)=0 \text { implies } p\left(v^{-1}(A)\right)=0 .
$$

Let $C$ be the continuity set of $v$. We then have

$$
\begin{aligned}
p\left(v^{-1}(A)\right) & =v\left(v^{-1}(A) \cap C\right) \\
& =m\left(v\left[v^{-1}(A) \cap C\right]\right) \quad[10, \text { Chapter III, (13.3) }] \\
& \leqslant m(A),
\end{aligned}
$$

and so (10).

Let us recall a few facts about (BV) functions from [3] and [4].

Suppose $F(t)$ is a real-valued (BV) function on $[0,1]$. This induces a signed measure $V_{F}$ and a nonnegative measure $v_{F}$ on $\mathscr{B}([0,1])$ in the usual way, viz. $V_{F}$ is determined by its values on intervals, $V_{F}((a, b])=F(b)-F(a)$, and $v_{F}=\left|V_{F}\right|=$ the "total variation measure" of $V_{F}$. In the special case of an (AC) function, these measures are given by

$$
\begin{aligned}
& V_{F}(B)=\int_{B} F^{\prime}(t) d t, \\
& v_{F}(B)=\int_{B}\left|F^{\prime}(t)\right| d t,
\end{aligned}
$$

the derivative $F^{\prime}$ existing a.e. In general, $V_{F}, v_{F}$ can be broken into atomic components and continuous (i.e. atomless) components as follows. Writing $\Delta F(t)$ $=F(t)-F(t-)$, the jump parts are, for $V_{F}$ and $v_{F}$, respectively,

$$
J_{F}(B)=\sum_{t \in B} \Delta F(t), \quad j_{F}(B)=\sum_{t \in B}|\Delta F(t)| ;
$$

the continuous component of $V_{F}$ is just $\Psi_{F}=V_{F}-J_{F}$, while that of $v_{F}$ has the more interesting representation $[3$, p. 177]

$$
\psi_{F}(B)=\int_{\mathbf{R}} \nu_{F}(u, B) d u
$$


where $\nu_{F}$ is the Banach indicatrix (\$3). A monotone class argument at (13) (start with $\left.g(s, u)=I_{\Gamma}(u) I_{B}(s)\right)$ shows [4]

$$
\int_{0}^{1} g(s, F(s)) \psi_{F}(d s)=\int_{\mathbf{R}} \int_{0}^{1} g(s, u) \nu_{F}(u, d s) d u .
$$

We now wish to calculate $\Psi, \psi$ for functions of the form $F=H \circ v$, where $v$ is as above (around (10)) and $H: \mathbf{R} \rightarrow \mathbf{R}$ is Lipschitz, which implies that $H$ is (AC) and $H \circ v$ is (BV). Recall that $p$ denotes the continuous component of $v$.

(15) Lemma. (a) $\Psi_{H \circ v}(B)=\int_{B} H^{\prime}(v(s)) p(d s)$,

(b) $\psi_{H \circ v}(B)=\int_{B}\left|H^{\prime}(v(s))\right| p(d s)$.

Proof. The derivative $H^{\prime}$ exists a.e., so that $H^{\prime}(v(s))$ is well defined $p$-a.e. in view of (10). Let us assume, for the moment, that $H$ is nondecreasing, and put $\hat{H}(u)=\inf \{y: H(y)>u\}$; this is the right continuous "inverse" of $H$. A little picture will show that, if $u$ is not one of the countable number of flat levels on the graph of $H$, then $\nu_{H} \circ v(u, B)=\nu_{v}(\hat{H}(u), B)$. Thus, applying (13) to $F=H \circ v$, which is nondecreasing, we have

$$
\begin{aligned}
\Psi_{H \circ v}(B) & =\psi_{H \circ v}(B)=\int_{\mathbf{R}} \nu_{v}(\hat{H}(u), B) d u \\
& =\int_{\mathbf{R}} \nu_{v}(y, B) H^{\prime}(y) d y \quad(y=\hat{H}(u)) \\
& =\int_{B} H^{\prime}(v(s)) p(d s),
\end{aligned}
$$

by (14) since $p$ is the continuous component of $v$. To prove (a) in the general case, we write $H$ as the difference of two monotone functions and use the above special case.

Finally, it is a standard fact that, since $v=|V|$, the continuous components stand in the same relation: $\psi=|\Psi|$; then (b) is a well-known consequence of (a).

Note. Although (15)(b) and (18') below are not needed for the proof of Theorem $A$, they are included to complete our results on functions of bounded variation and require almost no additional work.

We now extend these results to the vector-valued case. Thus $Z:[0,1] \rightarrow \mathbf{R}^{d}$ is (BV) with coordinates $\left(Z_{1}(t), \ldots, Z_{d}(t)\right)$, each $Z_{i}$ being (BV). We associate with $Z$ a vector-valued variation measure $V_{Z}(B)=\left(V_{1}(B), \ldots, V_{d}(B)\right)$ where $V_{i}=V_{Z_{i}}$ is determined as above. The jump and continuous components are given by

$$
J_{Z}(B)=\left(J_{1}(B), \ldots, J_{d}(B)\right), \quad \Psi_{Z}(B)=\left(\Psi_{1}(B), \ldots, \Psi_{d}(B)\right)
$$

with a self-explanatory notation. We note that $J_{Z}$ can also be written

$$
J_{Z}(B)=\sum_{t \in B} \Delta Z(t)
$$

The scalar variation measure $v_{z}$ is formed by analogy with the variation in the real-valued case: $v_{Z}([0, t])$ is the variation over $[0, t]$ computed by the usual "variation sums" $\Sigma\left|Z\left(t_{i+1}\right)-Z\left(t_{i}\right)\right|$, where the norms are taken in $\mathbf{R}^{d}$. This is given explicitly [3, p. 177] by

$$
v_{Z}(B)=\psi_{Z}(B)+j_{Z}(B)
$$


where the jump part

$$
j_{Z}(B)=\sum_{t \in B}|\Delta Z(t)|
$$

and the continuous part $\psi_{Z}$ is given by (7).

We remark that each coordinate function satisfies $v_{Z_{i}}(B) \leqslant v_{Z}(B)$ and likewise for the $\psi$ 's.

We now want to rewrite the vector variation measure in a more convenient form for later use. Only the continuous component requires attention. Let $v(t)=$ $v_{Z}([0, t])$, and recall that $\psi_{Z}$ is the continuous part of $v(t)$. According to 2.5.16 of [3], there is a Lipschitz function $G: \mathbf{R} \rightarrow \mathbf{R}^{d}$, with Lipschitz constant at most 1 , such that $Z=G \circ v$.

It now follows that

$$
\Psi_{Z}(B)=\int_{B} G^{\prime}(v(s)) \psi_{Z}(d s)
$$

The existence of the integral in (16) is an immediate consequence of (10), and then (16) itself is a direct application of (15) to the components $G=\left(G_{1}, \ldots, G_{d}\right)$ of $G$.

Here is the main result needed for Theorem A. Let $\mathscr{D}=\mathscr{D}\left(\mathbf{R}^{d}\right)$ be the space of infinitely differentiable real-valued functions on $\mathbf{R}^{d}$ with compact support. If $Z$ is (BV) and $f \in \mathscr{D}$, then $f \circ Z$ is again (BV), and we want representations of the signed and nonnegative variation measures of $f \circ Z$.

(17) LEMMA. Let $Z$ be $(B V)$ and $f \in \mathscr{D}$; then

$$
\begin{gathered}
V_{f \circ Z}(B)=\int_{B} \nabla f(Z(s)) \cdot \Psi_{Z}(d s)+J_{f \circ Z}(B), \\
v_{f \circ Z}(B)=\int_{\mathbf{R}^{d}} \int_{0}^{1}\left|\nabla f(z) \cdot G^{\prime}(v(s))\right| \nu_{Z}(z, d s) H^{1}(d z)+j_{f \circ Z}(B) .
\end{gathered}
$$

If $Z$ is saltus, (18) and $\left(18^{\prime}\right)$ hold with the integral terms deleted.

Note. If $F=\left(F_{1}, \ldots, F_{d}\right)$ is a function and $\Psi=\left(\Psi_{1}, \ldots, \Psi_{d}\right)$ is a measure, then $\int f \cdot d \Psi \equiv \Sigma_{i} \int F_{i} d \Psi_{i}$.

Proof. The jump terms are correct from the definitions.

To get (18), we will first apply (15)(a) to the function $H=f \circ G$, and then use (16). Recall that $Z=G \circ v$, and that $v=v_{Z}$, so that $p=\psi_{Z}$. Hence, since $H^{\prime}(y)=\nabla f(G(y)) \cdot G^{\prime}(y)$,

$$
\Psi_{f \circ Z}(B)=\Psi_{H \circ v}(B)=\int_{B} \nabla f(Z(s)) \cdot G^{\prime}(v(s)) \psi_{Z}(d s) .
$$

Putting $g(s)=1_{B}(s) \nabla f(Z(s))$, the integral above is

$$
\begin{aligned}
\sum_{i} \int g_{i}(s)\left(G^{\prime}(v(s))\right)_{i} \psi_{Z}(d s) & =\sum_{i} \int g_{i}(s)\left(\Psi_{Z}\right)_{i}(d s) \text { by (16) } \\
& =\int g \cdot d \Psi_{Z}
\end{aligned}
$$

which is the integral term in (18).

The proof of $\left(18^{\prime}\right)$ is similar, using (15)(b) and taking (7) into account. 
Let $Z=\left(Z_{1}, \ldots, Z_{d}\right)$ be saltus, so each $Z_{i}$ is also saltus. By a simple extension of [10, Chapter IV, §8], we have

$$
v_{Z}(B) \leqslant \sum_{i=1}^{d} v_{Z_{i}}(B)
$$

hence a similar relation for the $\psi$ 's. But, by Riesz and Sz.-Nagy [9, p. 14], $\psi_{Z_{i}}=0$ for each $i$. Thus $\psi_{Z}=0$ and so $\nu_{Z}(z,[0,1])=0$ for $H^{1}$-a.e. $z$, and the integral terms in (18), $\left(18^{\prime}\right)$ are zero.

The theorem applies more generally than to $f \in \mathscr{D}$, but this is the only case we shall use.

5. Proof of Theorem A. We begin with a heuristic proof of Theorem $A^{\prime}$ which indicates why a formula like (1) should give the local time of $X+Z$. Let $\phi_{t}(\Gamma)$ be the occupation measure of $X+Z$, so that the density, if one exists, will be given a.e. by

$$
\begin{aligned}
\gamma_{t}(x) & =\frac{d}{d x} \phi_{t}((-\infty, x])=\frac{d}{d x} \int_{0}^{t} I_{(-\infty, x]}(X(s)+Z(s)) d s \\
& =\int_{-\infty}^{\infty} \int_{0}^{t} \frac{d}{d x} I_{[y+Z(s), \infty)}(x) \alpha(y, d s) d y \quad(\text { by }(5)) \\
& =\int_{-\infty}^{\infty} \int_{0}^{t} \delta(x-y-Z(s)) \alpha(y, d s) d y \\
& =\int_{0}^{t} d_{s} \alpha_{s}(x-Z(s))+\int_{0}^{t} \alpha_{s}^{\prime}(x-Z(s))(d Z / d s) d s \\
& =\alpha_{t}(x-Z(t))+\int_{0}^{t} \alpha_{s}^{\prime}(x-Z(s))(d Z / d s) d s,
\end{aligned}
$$

which is (1).

To prove Theorem A it suffices to establish

$$
\int_{0}^{t} f(X(s)+Z(s)) d s=\int_{\mathbf{R}^{d}} \gamma_{t}(x) f(x) d x, \quad 0 \leqslant t<1, \quad f \in \mathscr{D},
$$

where $\gamma$ is given by (8). Indeed, (19) then extends to all nonnegative Borel functions $f$. The choice $f=I_{\Gamma}, \Gamma \in \mathscr{B}_{d}$, then shows that $\gamma_{t}$ is the density of the occupation measure $\phi_{t}$ of $X+Z$.

For simplicity we consider only $t=1$. Let $f \in \mathcal{D}$. Using $g(s, x)=f(x+Z(s))$ in (5), we have

$$
\begin{aligned}
\int_{0}^{1} f(X(s)+Z(s)) d s & =\int_{\mathbf{R}^{d}} \int_{0}^{1} f(x+Z(s)) \alpha(x, d s) d x \\
& =\int_{\mathbf{R}^{d}}\left[f(x+Z(1)) \alpha_{1}(x)-\int_{0}^{1} \alpha_{s}(x) d_{s} f(x+Z(s))\right] d x,
\end{aligned}
$$

the term in brackets resulting from a standard integration by parts (Saks [10, III, $\S 14])$. We split the integral on the right into two pieces, the first of which is

$$
\int_{\mathbf{R}^{d}} f(x) \alpha_{1}(x-Z(1)) d x
$$


and the second (except for the minus sign)

$$
\int_{\mathbf{R}^{d}} \int_{0}^{1} \alpha_{s}(x) d_{s} f(x+Z(s)) d x .
$$

According to (18), the inner integral in (21) equals

$$
\int_{0}^{1} \alpha_{s}(x) \nabla f(x+Z(s)) \cdot \Psi_{Z}(d s)+\sum_{s} \alpha_{s}(x)\{f(x+Z(s))-f(x+Z(s-))\} .
$$

The jump term here is majorized by $\alpha_{1}(x)\|\nabla f\|_{\infty} j_{Z}([0,1])$, which is in $L^{1}\left(\mathbf{R}^{d}\right)$. Using condition (c) in the statement of the theorem, we obtain, upon integrating this term over $\mathbf{R}^{d}$, the value

$$
\int_{\mathbf{R}^{d}} f(x) \sum_{s}\left(\alpha_{s}(x-Z(s))-\alpha_{s}(x-Z(s-))\right) d x .
$$

Similarly, $\alpha_{s}(x) \nabla f(x+Z(s))$ is $\psi_{Z_{i}} \times m_{d}$-integrable for $i=1, \ldots, d$, so that we may integrate the dot product integral over $\mathbf{R}^{d}$. Thus we find the following evaluation of (21):

$$
\begin{aligned}
\int_{\mathbf{R}^{d}}\left[\int_{0}^{1} \alpha_{s}(x-Z(s)) \nabla f(x)\right. & \cdot \Psi_{Z}(d s) \\
& \left.+f(x) \sum_{s}\left(\alpha_{s}(x-Z(s))-\alpha_{s}(x-Z(s-))\right)\right] d x .
\end{aligned}
$$

To untangle the first term here, we expand the dot product integral thus:

$$
\begin{aligned}
\int_{\mathbf{R}^{d}} \sum_{j=1}^{d} \int_{0}^{1} \alpha_{s}(x-Z(s)) \frac{\partial f}{\partial x_{j}} \Psi_{j}(d s) d x \\
=-\int_{\mathbf{R}^{d}} f(x) \sum_{j=1}^{d} \int_{0}^{1} \frac{\partial \alpha_{s}(x-Z(s))}{\partial x_{j}} \Psi_{j}(d s) d x \\
=-\int_{\mathbf{R}^{d}} f(x) \int_{0}^{1} \nabla \alpha_{s}(x-Z(s)) \cdot \Psi_{Z}(d s) d x
\end{aligned}
$$

It should be remarked that the joint continuity of $\alpha_{s}(x)$ guarantees the joint measurability of the partial derivatives of $\alpha$; see Saks [10, Chapter V, (4.1)]. Putting everything together, we get (19), and so the first assertion of Theorem $A$ and formula (8), viz.

$$
\begin{aligned}
\gamma_{t}(x)= & \alpha_{t}(x-Z(t))+\int_{0}^{t} \nabla \alpha_{s}(x-Z(s)) \cdot \Psi_{Z}(d s) \\
& -\sum_{s<t}\left[\alpha_{s}(x-Z(s))-\alpha_{s}(x-Z(s-))\right] .
\end{aligned}
$$

Looking at (8) again, the integral term is obviously continuous in $t$ for a.e. $x$. Taking left limits at $t$ in the remaining terms we find

$$
\alpha_{t}(x-Z(t-))-\sum_{s<t}\left[\alpha_{s}(x-Z(s))-\alpha_{s}(x-Z(s-))\right] .
$$

If we now add and subtract $\alpha_{t}(x-Z(t))$ we obtain $\alpha_{t-}(x)=\alpha_{t}(x)$. The proof of Theorem $\mathbf{A}$ is now complete. 
It is clear that under some more restrictions, the new local time $\gamma$ will be continuous in $x$ as well. For example, this will be so if $Z$ is continuous and $\nabla \alpha_{s}(\cdot)$ is continuous.

6. Perturbation by a saltus function. By a slight modification of the argument in $\S 5$, taking into account the last statement of (17), we have

Theorem C. Suppose $Z(t)$ is a saltus function and $\alpha$ is subject only to condition (c) of Theorem A, viz.

(c) $\sum_{s}\left|\alpha_{s}(x-Z(s))-\alpha_{s}(x-Z(s-))\right|$ is in $L^{1}\left(\mathbf{R}^{d}\right)$.

Then $X(t)+Z(t)$ has a local time and (8) holds (with the integral term deleted).

A sufficient condition for (c), often encountered in the context of stochastic processes, is that $\alpha$ satisfy a uniform Hölder condition of some order $\beta$ in its space variable $x$ and that the jumps of $Z$ be summable to the power $\beta$. Thus, for example, in the case of Brownian motion, we have this situation for every $\beta<\frac{1}{2}$ by Corollary 2 of [6]. This affords a slight strengthening of Meyer's theorem ( $\$ 1)$ in a special case: if $X_{t}$ is Brownian motion and $Z_{t}$ is an arbitrary process whose trajectories are saltus functions with $\beta$-summable jumps for some $\beta<\frac{1}{2}$, then a.s. $X_{t}+Z_{t}$ has a local time (given in accordance with Theorem $\mathrm{C}$ ). We note that $\beta$ may depend on the trajectory, and that $Z_{t}$ need not be an adapted process.

A simple case of this type of result, in which $Z$ is a step function with a finite number of jumps, was used in $[5, \S 0]$ to give a trivial proof of the following theorem of Getoor and Kesten [6]: a real-valued process $X_{t}$ with stationary independent increments, having either a Gaussian component or a Lévy measure with infinite mass around the origin, has a (jointly continuous) local time with probability 0 or 1 .

7. Other combinations. The reader will have no trouble in formulating and proving, by the same methods, an analogous theorem for other combinations of $X(t)$ and $Z(t)$. Qualitatively the result may be stated thus: let $\phi(x, z)$ be any "reasonable" measurable function; then, for $X, Z$ as in Theorem $A$, the function $\phi(X(t), Z(t))$ has a local time and (3) holds. Some cases can be immediately reduced to the case of addition; for example, if $\phi(x, z)=x z$, one looks at $\exp (\ln X(t)+\ln Z(t))$.

Rather than pursue this general question, we will consider a specific example. Let $X:[0,1] \rightarrow \mathbf{R}$ be continuous and satisfy the conditions of Theorem A. Put $Z(t)=\max _{0 \leqslant s \leqslant t} X(s)$, the "progressive maximum" of $X: Z(t)$ is a continuous, monotone increasing, and so (BV) function. In [6, §12], we considered the function $Z(t)-X(t)$ and compared this with the well-known case of Brownian motion $X(t)$, where $Z-X$ has the same probability law as $|X|$.

Instead, let us look at $X(t) / Z(t)$ for $t>0$. The local time $\gamma_{t}(x)$ is, in this case,

$$
\gamma_{t}(x)=\alpha_{t}(x Z(t)) Z(t)-x \int_{0}^{t} \alpha_{s}^{\prime}(x Z(s)) Z(s) Z(d s)-\int_{0}^{t} \alpha_{s}(x Z(s)) Z(d s) .
$$

For values of $x$ near 1 , this gives a new measure of the "record values" of $X$. 
8. Probabilistic examples; Proof of Theorem D. We present an example of a class of real-valued functions $X(t)$ having a local time $\alpha_{t}(x)$ which is jointly continuous, such that $\alpha_{t}(\cdot)$ is (AC) for every $t$, and such that $\alpha_{t}^{\prime}(x)=\partial \alpha_{t}(x) / \partial x$ is jointly continuous as well as integrable over $[0,1] \times \mathbf{R}$.

Let $(\Omega, \mathcal{F}, \mathbf{P})$ be a probability space, $\mathbf{E}$ denoting the corresponding expectation. Our example will consist of a Gaussian random process of which almost every trajectory fits the above description.

We begin with a Kolmogorov-type lemma. Let $D$ be the set of dyadic rationals in $[0,1]$.

(22) LEMMA. For each $r, s \in D$, let $Y_{r s}$ be a nonnegative random variable subject to the following assumptions:

(a) There exist positive functions $\eta(\delta), \varepsilon(\delta)$ such that

$$
\begin{gathered}
\sum_{m} \varepsilon\left(2^{-m}\right)<\infty, \quad \sum_{m} 2^{m} \eta\left(2^{-m}\right)<\infty, \\
\mathbf{P}\left\{Y_{r s} \geqslant \varepsilon(|r-s|)\right\} \leqslant \eta(|r-s|),
\end{gathered}
$$

(b) for any $u, v, w \in D, Y_{u w} \leqslant Y_{u v}+Y_{v w}$,

(c) $Y_{u v}=Y_{v u}, Y_{u u}=0$;

then a.s., $Y_{r s} \rightarrow 0$ as $|r-s| \rightarrow 0$.

This is a standard result for functions of one variable. For the present case a proof may be modeled on that of Proposition III-5-2 (b) in Neveu's book [8], taking $Z_{m}=\max _{0 \leqslant k<2^{m}} Y_{k 2^{-m},(k+1) 2^{-m}}$ in Neveu's proof.

As a special case, suppose

$$
\mathbf{E}\left(Y_{r s}^{2}\right) \leqslant c|r-s|^{1+\beta} \quad(\beta>0) .
$$

The above conditions will be satisfied if we take $\varepsilon(\delta)=\delta^{\rho}$, where $0<\rho<\beta / 2$, and $\eta(\delta)=\delta^{1+\beta-2 \rho}$.

(24) LEMMA. Let $X(t)$ be a nonrandom function having a jointly continuous local time $\alpha_{t}(x)$ such that $\alpha_{t}(\cdot)$ is $(A C)$ for each $t \in D$. Assume, moreover, that $\alpha_{t}^{\prime} \in$ $L^{2}(d x)$ for each $t \in D$ and that $\alpha_{t_{n}}^{\prime}$ is Cauchy in $L^{2}(d x)$ whenever $t_{n}$ is Cauchy in $D$. Then $\alpha_{t}(\cdot)$ is $(A C)$ for every $t \in[0,1]$.

Let $t \in[0,1]$ and choose $t_{n} \in D, t_{n} \rightarrow t$. We then have the existence of $\lim _{n} \alpha_{t_{n}}^{\prime}(x)=\beta(x)$, say, in the sense of $L^{2}(d x)$, and also in $L^{1}$ on any finite $x$-interval. Thus, as $n \rightarrow \infty$,

$$
\alpha_{t_{n}}(x)=\alpha_{t_{n}}(0)+\int_{0}^{x} \alpha_{t_{n}}^{\prime}(y) d y
$$

becomes

$$
\alpha_{t}(x)=\alpha_{t}(0)+\int_{0}^{x} \beta(y) d y
$$

whence (24).

Now let $X_{t}, 0 \leqslant t \leqslant 1$, be a mean 0 , Gaussian random process on $(\Omega, \mathcal{F}, \mathbf{P})$, having stationary increments. This implies that the incremental variance $\sigma^{2}(s, t)=$ $\mathbf{E}\left(X_{s}-X_{t}\right)^{2}$ is a function of $|s-t|$ only. In particular, if $\sigma^{2}(s, t) \sim K|s-t|^{\gamma}$, we 
have the so-called (in [1]) index $\gamma$ processes. Berman [1] has shown that, if

$$
\int_{0}^{1} \int_{0}^{1} \sigma^{-(2 p+1)}(u, v) d u d v<\infty
$$

for $p>\frac{1}{2}$, then, a.s., the trajectory $X_{t}$ has a jointly continuous local time $\alpha_{t}(x)$, and that, for each $t$, the "space" derivatives $\alpha_{t}^{\prime}(x), \alpha_{t}^{\prime \prime}(x), \ldots, \alpha_{t}^{[p]}(x)$ will exist, and belong to $L^{2}(d x)$, a.s. It also follows from simple real-variable considerations that each of these derivatives up to order $[p]-1$ is (AC). By an obvious argument, we may assume that, a.s., these properties hold simultaneously for every $t \in D$.

Let

$$
\hat{\alpha}_{t}(\theta)=\int_{-\infty}^{\infty} e^{i x \theta} \alpha_{t}(x) d x=\int_{0}^{t} e^{i \theta X} d s
$$

be the Fourier transform of $\alpha_{t}(x)$; the second equation follows from (5). Of course, $\hat{\alpha}_{t}(\theta)$ is the Fourier-Stieltjes transform (alias characteristic function) of the occupation measure $\mu_{t}$.

We now turn to the proof of Theorem $D(\S 1)$, which says in effect that the index $\gamma$ process furnishes the desired example.

Define

$$
Y_{r s}^{(p)}=\left(\int_{-\infty}^{\infty}|\theta|^{2 p}\left|\hat{\alpha}_{r}(\theta)-\hat{\alpha}_{s}(\theta)\right|^{2} d \theta\right)^{1 / 2}, \quad r, s \in D ;
$$

conditions (b) and (c) of (22) are easily verified, and a standard calculation shows

$$
\mathbf{E}\left(Y_{r s}^{(p)}\right)^{2}=K \int_{r}^{s} \int_{r}^{s} \sigma^{-(2 p+1)}(u, v) d u d v, \quad r<s
$$

for some constant $K$, independent of $r, s$. (If $p$ is an integer, then, by standard Fourier analysis,

$$
Y_{r s}^{(p)}=\left\|\alpha_{r}^{(p)}-\alpha_{s}^{(p)}\right\|_{2}
$$

the norm referring to $L^{2}(d x)$.) In the index $\gamma$ case, the integral in (27) will be $\sim K^{\prime}|s-r|^{1+(1-\gamma(p+1 / 2))}$, so that (23) will hold for $Y_{r s}^{(p)}$ whenever

$$
\gamma<2 /(2 p+1)
$$

and then (22) will be in force for $Y^{(p)}$.

Suppose, first, that $p=1, \gamma<\frac{2}{3}$; then, for almost every trajectory, $\alpha_{t_{n}}^{\prime}(x)$ will be Cauchy in $L^{2}(d x)$ whenever $t_{n}$ is Cauchy in $D$. By (24), $\alpha_{t}(\cdot)$ is $(A C)$ for every $t \in[0,1]$.

We now note that, if $\gamma<\frac{1}{2}$, (28), and so (25), will hold for $p>\frac{3}{2}$. Thus, a.s.,

$$
\int_{-\infty}^{\infty}|\theta|^{2 p}\left|\hat{\alpha}_{r}(\theta)-\hat{\alpha}_{s}(\theta)\right|^{2} d \theta \rightarrow 0 \text { as }|r-s| \rightarrow 0,
$$

for $r, s \in D$. This says that $|\theta|^{p} \hat{\alpha}_{r}(\theta)$ is Cauchy in $L^{2}(d \theta)$ whenever $r$ runs through a Cauchy sequence in $D$. If $r \rightarrow t \in[0,1]$, then (26) shows that the (pointwise and $\left.L^{2}\right)$ limit must be $|\theta|^{p} \hat{\alpha}_{t}(\theta)$. It follows that (29) holds for arbitrary $r, s \in[0,1]$ : if not, there exist $0 \leqslant u_{n}, v_{n} \leqslant 1$ and $\varepsilon>0$ such that $\left|u_{n}-v_{n}\right| \rightarrow 0$ but $Y_{u_{n}, v_{n}}>\varepsilon$. By taking subsequences, we may assume $u_{n} \rightarrow t, v_{n} \rightarrow t$. Choose $r_{n}, s_{n} \in D$ as follows: $\left|r_{n}-u_{n}\right|<2^{-n},\left|s_{n}-v_{n}\right|<2^{-n}, Y_{u_{n}, r_{n}}<\varepsilon / 3, Y_{v_{n}, s_{n}}<\varepsilon / 3$. The possibility of such a choice is left to the reader to prove. Property (b) in (22) finishes the argument. 
Consider now a fixed trajectory such that $\alpha_{t}(\cdot)$ is (AC) for every $t \in[0,1]$ and for which (29) holds. We are assuming the process is of index $\gamma<\frac{2}{3}$ so that almost every trajectory has these properties.

We observe first that $\theta \hat{\alpha}_{t}(\theta)$ is in $L^{1}(d \theta)$ :

$$
\int_{-\infty}^{\infty}|\theta|\left|\hat{\alpha}_{t}(\theta)\right| d \theta \leqslant \int_{|\theta|<1}+\int_{|\theta|>1} .
$$

The first term is obviously finite. As for the second, it equals

$$
\begin{aligned}
& \int_{|\theta|>1}|\theta|^{1-p}|\theta|^{p}\left|\hat{\alpha}_{t}(\theta)\right| d \theta \\
& \leqslant\left(\int_{|\theta|>1}|\theta|^{2-2 p} d \theta\right)^{1 / 2}\left(\int_{|\theta|>1}|\theta|^{2 p}\left|\hat{\alpha}_{t}(\theta)\right|^{2} d \theta\right)^{1 / 2}
\end{aligned}
$$

of which the second factor is finite from our earlier work, and the first is finite as long as $p>\frac{3}{2}$. By the Fourier inversion theorem,

$$
\alpha_{t}^{\prime}(x)=-\frac{i}{2 \pi} \int_{-\infty}^{\infty} e^{-i \theta x} \theta \hat{\alpha}_{t}(\theta) d \theta
$$

holds for a.e. $x$; however, we may alter the derivative so that (31) holds for all $x$. We now show that the version of $\alpha_{t}^{\prime}(x)$ given by (31) is jointly continuous. Of course the continuity in $x$ alone is almost obvious.

Let us consider

$$
\begin{aligned}
\alpha_{s}^{\prime}(x)-\alpha_{t}^{\prime}(y)=- & \frac{i}{2 \pi} \int_{-\infty}^{\infty} e^{-i \theta x}\left(\hat{\alpha}_{s}(\theta)-\hat{\alpha}_{t}(\theta)\right) d \theta \\
& \quad-\frac{i}{2 \pi} \int_{-\infty}^{\infty} \theta \hat{\alpha}_{t}(\theta)\left(e^{-i \theta x}-e^{-i \theta y}\right) d \theta \\
\equiv & A+B .
\end{aligned}
$$

Dropping the nuisance factor $-i / 2 \pi$, and writing $f_{s t}(\theta)=\hat{\alpha}_{s}(\theta)-\hat{\alpha}_{t}(\theta)$, we have

$$
|A| \leqslant \int_{-\infty}^{\infty}|\theta|\left|f_{s t}(\theta)\right| d \theta=\int_{|\theta|<1}+\int_{|\theta|>1} .
$$

The first term tends to 0 as $|s-t| \rightarrow 0$ by dominated convergence since $f_{s t}(\theta) \rightarrow 0$ for each $\theta$. The second term is treated as in (30), appealing to (29). Thus $A \rightarrow 0$ as $|s-t| \rightarrow 0$.

Now fix $(t, y)$ and let $(s, x) \rightarrow(t, y)$. Then $A \rightarrow 0$ as just noted, and $B \rightarrow 0$ by dominated convergence.

\section{REFERENCES}

1. S. Berman, Harmonic analysis of local times and sample functions of Gaussian processes, Trans. Amer. Math. Soc. 143 (1969), 269-281.

2. A. Bruckner and J. Ceder, On the sum of Darboux functions, Proc. Amer. Math. Soc. 51 (1975), 97-102.

3. H. Federer, Geometric measure theory, Springer-Verlag, Berlin and New York, 1969.

4. D. Geman and J. Horowitz, Occupation times for functions with countable level sets and the regeneration of stationary processes, Z. Wahrsch. Verw. Gebeite 35 (1976), 189-211.

5. , Occupation densities, Ann. Probab. 8 (1980), 1-67. 
6. R. K. Getoor and H. Kesten, Continuity of local times for Markov processes, Comp. Math. 24 (1972), 277-303.

7. P. A. Meyer, Un cours sur les intégrales stochastiques, Sem. de Probabilitiés X, Lecture Notes in Math., vol. 511, Springer-Verlag, Berlin and New York, 1976.

8. J. Neveu, Bases mathématiques du calcul des probabilités, Masson, Paris, 1964.

9. F. Riesz and B. Sz.-Nagy, Functional analysis, Ungar, New York, 1955.

10. S. Saks, Theory of the integral, Dover, New York, 1964 (reprint).

11. J. Smital, On the sum of continuous and Darboux functions, Proc. Amer. Math. Soc. 60 (1976), 183-184.

Department of Mathematics, University of Massachusetts, Amherst, Massachusetts 01003 